\title{
Predicting large solar flares with data assimilation
}

\author{
Antoine Strugarek and Paul Charbonneau \\ Université de Montréal, C.P. 6128 Succ. Centre-Ville, Montréal, QC H3C-3J7, Canada \\ email: strugarek@astro.umontreal.ca
}

\begin{abstract}
We propose to use a deterministically-driven class of self-organized criticality sandpile models to carry out predictions of the largest, most dangerous, and hardest to predict solar flares.
\end{abstract}

Solar and stellar flares are magnetically-driven, scale-invariant energy release events spanning over 8 orders of magnitude in energy (Aschwanden et al. 2014). The prediction of the largest solar flares, of class $\mathrm{X}$, is a particularly hard task due the scarcity of such events. The detailed 3D modelling of flaring active regions still requires today too much numerical resources to be routinely used for near real-time predictions. Alternative, empirical models hence have to be designed to perform such predictions. Among the models that adequately reproduce the power-law distribution in flare sizes, avalanche models (Lu \& Hamilton 1991) have the advantage of being numerically cheap to operate. However, they usually rely on a stochastic driver, which can be expected to degrade their predictive capabilities. Building on the pioneering work of Lu and Hamilton, we developed a class of avalanche models which succeed in minimizing the built-in stochastic ingredients while retaining the solar flares power-law distribution (Strugarek et al. 2014). We showed that the largest avalanches occurring in these models are robust with respect to the stochastic realization (Strugarek \& Charbonneau 2014), which opens new perspectives for the prediction of the largest (and most dangerous) solar flares.

Our ultimate goal is to combine data assimilation of the GOES X-ray flux with our avalanche models to carry out actual predictions. We transformed the GOES X-ray flux into a series of peaks that is fed to our model, which automatically finds an initial condition that is compatible with the observed series of events. The initial condition is found using non-gradient based optimization techniques, due to the high sensitivity of the avalanche model to slight alterations of the initial conditions (said differently, the cost-function associated to the minimization process has a lot of local minima). The costfunction is designed to be versatile: depending on the user needs, different weights can be given to matches, missed, and false alarms events. Preliminary tests demonstrated the possibility to use such data assimilation procedure with real data. In a near future we will test our prediction model against a significant sample of past GOES large events, and study the possibility to use our data assimilation package in near real-time applications.

\section{References}

Aschwanden, M. J., Crosby, N. B., Dimitropoulou, M., et al. 2014, Space Sci. Rev., 29

Lu, E. T. \& Hamilton, R. J. 1991, ApJL, 380, L89

Strugarek, A. \& Charbonneau, P. 2014, Sol. Phys., 289, 4137

Strugarek, A., Charbonneau, P., Joseph, R., \& Pirot, D. 2014, Sol. Phys., 289, 2993 